\title{
PLASTIC STRESSES INDUCED BY A RIGID RING EMBEDDED IN A THIN ANISOTROPIC PLATE UNDER UNIFORM TENSION*
}

\author{
BY \\ DAVID DURBAN ${ }^{1}$ \\ Engineering Department, Cambridge University, England
}

Introduction. The presence of a rigid fastening ring (Fig. 1) in a thin infinite sheet, subjected to remote uniform tension $\sigma_{\infty}$, is expected to affect the equibiaxial stress field in the vicinity of the rigid boundary. Here we present an analytical solution to this problem for pure power-hardening plastic materials, with transverse plastic anisotropy, modeled by

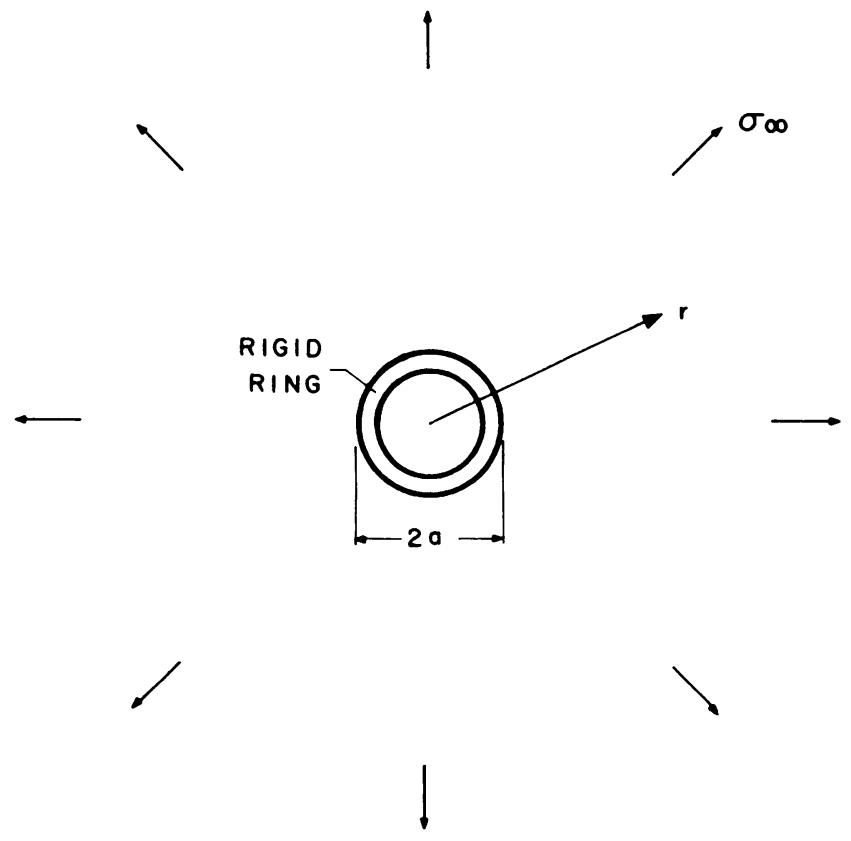

FIG. 1. A rigid ring is embedded in an infinite sheet subjected to remote uniform tension $\sigma_{\infty}$. The radial coordinate is denoted by $r$ and the rigid boundary is at $r=a$.

*Received May 29, 1986.

${ }^{1}$ On sabbatical leave (until October 1987). Permanent address: Technion-Israel Institute of Technology, Haifa, Israel. 
a theory proposed by Hill [1]. The effective stress $\sigma_{e}$ is defined as

$$
2(1+R) \sigma_{e}^{m}=(1+2 R)\left|\sigma_{1}-\sigma_{2}\right|^{m}+\left|\sigma_{1}+\sigma_{2}\right|^{m}
$$

where $\left(\sigma_{1}, \sigma_{2}\right)$ are the in-plane principal stresses, and parameters $(m, R)$ characterize the normal plastic anisotropy of the sheet. For the problem considered here we may identify the principal stresses with the polar components $\left(\sigma_{r}, \sigma_{\theta}\right)$. The dependence of the yield locus (1) on parameters $(m, R)$ is illustrated in Fig. 2 for the relevant quadrant where both stress components are positive. Note that the standard Mises and Tresca loci are obtained with ( $m=2, R=1)$ and ( $m=1, R=0)$, respectively.

The stress concentration problem, for a rigid circular inclusion, has been solved by Yang [2] using an earlier anisotropic theory proposed by Hill in [3]. That theory is just a particular case of (1) when $m=2$. A complete elasto/plastic solution to the problem, for

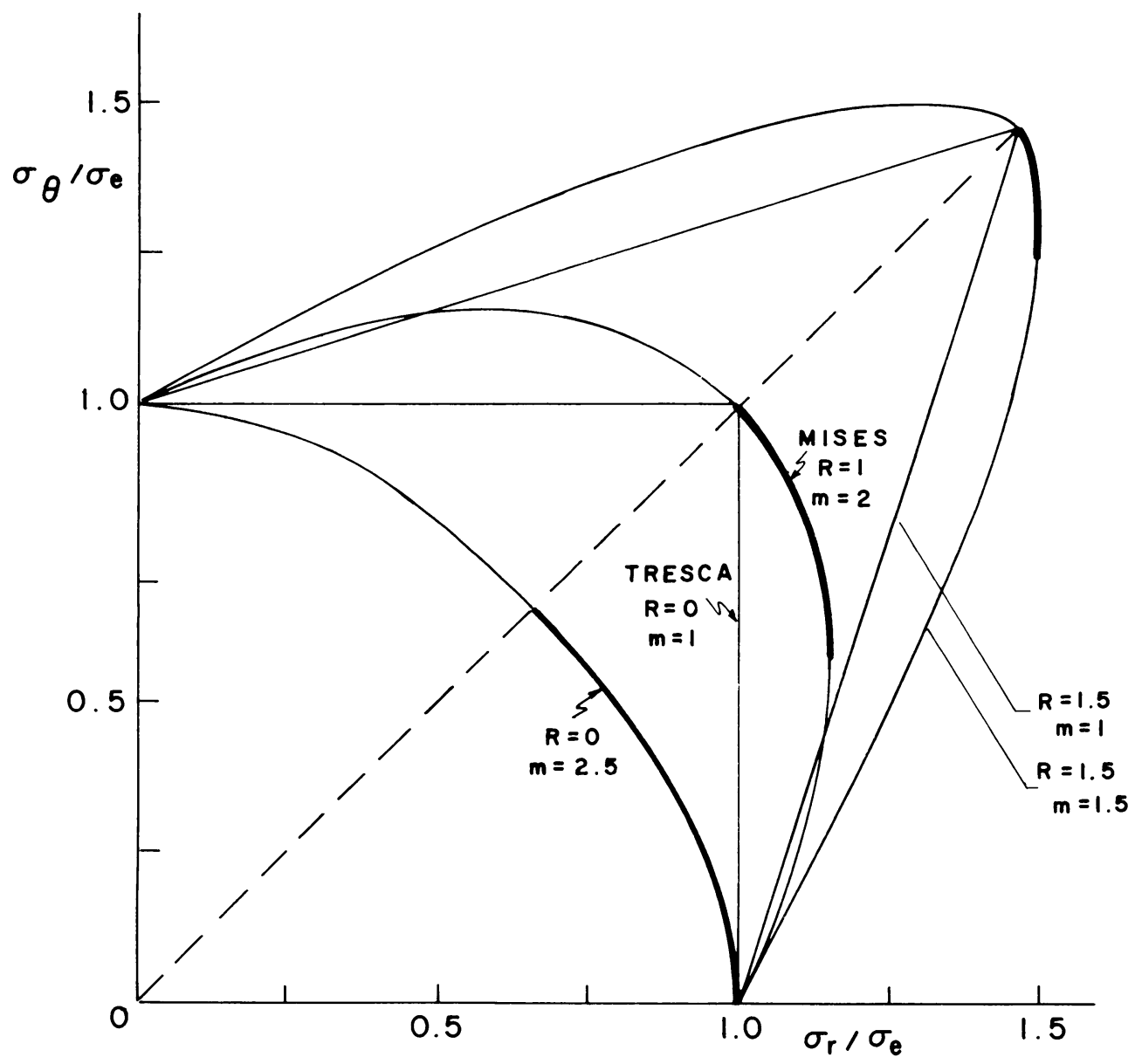

FIG. 2. Variation of the yield locus with parameters $(m, R)$ when both stress components are positive. The Mises material is described by $(m=2, R=1)$, and the Tresca material is described by $(m=1, R=0)$. The heavy lines show the operative parts of the yield locus. With $m=1$ only the point $\sigma_{r}=\sigma_{\theta}=\sigma_{\infty}=$ $(1+R) \sigma_{e}$ is operative. 
a restricted version of (1) with $m=1$, and accounting for linear strain hardening, has been given recently in [4].

The analogous problem of the stress concentration at a circular hole has been treated in several papers [5-8]. The analysis by Budiansky [7] contains a general investigation with different families of constitutive relations accounting for plastic anisotropy.

The solution in this paper is within the usual framework of axially-symmetric, planestress, small-strain plasticity. Elastic strains are neglected and changes in the thickness at the rigid boundary are permitted. Contact is made with the earlier result given in [2] for $m=2$.

Analysis. Anticipating that $\sigma_{r}>\sigma_{\theta}>0$ throughout the entire field, we rewrite definition (1) in the form

$$
2(1+R) \sigma_{e}^{m}=(1+2 R)\left(\sigma_{r}-\sigma_{\theta}\right)^{m}+\left(\sigma_{r}+\sigma_{\theta}\right)^{m} .
$$

Relation (2) is identically satisfied by the parametric representation

where

$$
\begin{aligned}
& \sigma_{r}-\sigma_{\theta}=J(\sin \alpha)^{2 / m} \sigma_{e}, \\
& \sigma_{r}+\sigma_{\theta}=H(\cos \alpha)^{2 / m} \sigma_{e},
\end{aligned}
$$

$$
H=(2+2 R)^{1 / m}, \quad J=\left(\frac{2+2 R}{1+2 R}\right)^{1 / m} .
$$

The stress components are therefore given by

$$
\sigma_{r}=S_{r} \sigma_{e}, \quad \sigma_{\theta}=S_{\theta} \sigma_{e},
$$

with

$$
\begin{aligned}
& S_{r}=\frac{1}{2}\left[H(\cos \alpha)^{2 / m}+J(\sin \alpha)^{2 / m}\right], \\
& S_{\theta}=\frac{1}{2}\left[H(\cos \alpha)^{2 / m}-J(\sin \alpha)^{2 / m}\right] .
\end{aligned}
$$

For the class of materials considered here it is possible to integrate the constitutive relations of the flow theory associated with (1). This leads to the deformation theory-type constitutive relations

$$
\varepsilon_{r}=\Gamma_{r} \varepsilon, \quad \varepsilon_{\theta}=\Gamma_{\theta} \varepsilon
$$

where $\left(\varepsilon_{r}, \varepsilon_{\theta}\right)$ are the usual strain components,

$$
\begin{aligned}
& \Gamma_{r}=\frac{1}{H}(\cos \alpha)^{2(m-1) / m}+\frac{1}{J}(\sin \alpha)^{2(m-1) / m}, \\
& \Gamma_{\theta}=\frac{1}{H}(\cos \alpha)^{2(m-1) / m}-\frac{1}{J}(\sin \alpha)^{2(m-1) / m},
\end{aligned}
$$

and $\varepsilon$ is the effective plastic strain determined by the uniaxial characteristic

$$
\varepsilon=\left(\sigma_{e} / \sigma_{0}\right)^{n}
$$

where $\sigma_{0}$ and $n$ are material constants.

With $u$ denoting the radial displacement, we have the kinematical relations

$$
\varepsilon_{r}=d u / d r, \quad \varepsilon_{\theta}=u / r
$$

which may be conjoined to form the compatibility equation

$$
r d \varepsilon_{\theta} / d r+\varepsilon_{\theta}-\varepsilon_{r}=0 .
$$


Now, we combine the equation of equilibrium

$$
r d \sigma_{r} / d r+\sigma_{r}-\sigma_{\theta}=0
$$

with (11) and eliminate the radial coordinate, thus obtaining the differential relation

$$
\left(\sigma_{r}-\sigma_{\theta}\right) d \varepsilon_{\theta}+\left(\varepsilon_{r}-\varepsilon_{\theta}\right) d \sigma_{r}=0 .
$$

Substituting in (13) the stresses from (5) and the strains from (7) gives, with the aid of (9),

$$
d \sigma_{e} / \sigma_{e}=f(\alpha) d \alpha,
$$

where

$$
f(\alpha)=-\frac{\left(S_{r}-S_{\theta}\right) \Gamma_{\theta}^{\prime}+\left(\Gamma_{r}-\Gamma_{\theta}\right) S_{r}^{\prime}}{\left(S_{r}-S_{\theta}\right) n \Gamma_{\theta}+\left(\Gamma_{r}-\Gamma_{\theta}\right) S_{r}}
$$

and the prime denotes differentiation with respect to $\alpha$. A further substitution of (6) and (8) results in

$$
f(\alpha)=\left(\frac{2}{m}\right) \frac{(m-1) \tan \alpha+(m-2) I(\tan \alpha)^{(m-2) / m}+I^{2}(\tan \alpha)^{(3 m-4) / m}}{n-(n-1) I(\tan \alpha)^{(2 m-2) / m}+I^{2}(\tan \alpha)^{(2 m-4) / m}},
$$

where

$$
I=H / J=(1+2 R)^{1 / m}
$$

The integral of Eq. (14) provides the solution of the problem; once the dependence of $\sigma_{e}$ on $\alpha$ has been determined, the corresponding expressions for the stresses and the strains follow from (5) and (7). The spatial profiles of these quantities can be found by transforming, from parameter $\alpha$ to the radial coordinate $r$, through the relation

$$
\frac{d r}{r}=-\frac{S_{r}^{\prime}+S_{r} f(\alpha)}{S_{r}-S_{\theta}} d \alpha
$$

which is obtained from (12) with the aid of (5) and (14).

In order to solve (14) we need to know the boundary data. At infinity, where $\sigma_{r}=\sigma_{\theta}=\sigma_{\infty}$, we have from (3a) that $\alpha=0$, while from (1) we find that $\sigma_{e}=(2 / H) \sigma_{\infty}$. At the inner boundary, where $u=0$, the circumferential strain $\varepsilon_{\theta}$ has to vanish or, from $(8 b)$,

$$
\tan \alpha_{a}=I^{-m /(2 m-2)} .
$$

Thus, parameter $\alpha$ varies from zero at infinity to the value given by (19) at $r=a$. The stress points on the yield locus will therefore move along the corresponding heavy parts shown in Fig. 2. Note, however, that with $m=1$ parameter $\alpha$ is identically equal to zero over the entire field, and the only operative part of the yield locus is the corner point $\sigma_{r}=\sigma_{\theta}=\sigma_{\infty}=(1+R) \sigma_{e}$. The solution for this special case is different from the one given by (14), as will be described shortly.

A convenient measure of the stress field near the rigid ring is given by the stress concentration factor, defined as

$$
k=\sigma_{e}(r=a) / \sigma_{\infty} .
$$

Thus, integration of (14) from infinity to the inner boundary gives

$$
k=\left(\frac{2}{H}\right) \exp \int_{0}^{\alpha_{a}} f(\alpha) d \alpha .
$$


Illustrative numerical results obtained from (21) are shown in Fig. 3. Also shown are the reference curves $k=2 / H$, which give the ratio $\sigma_{e} / \sigma_{\infty}$ for a uniform sheet under equibiaxial tension. Note that this is also the asymptotic value of (21) as $n$ becomes very large. It may be concluded from Fig. 3 that the $n$-sensitivity of the local stress field near the ring increases with parameter $m$ but decreases with parameter $R$.

When $m=2$, definition (1) and its associated constitutive relations are reduced to the earlier anisotropic theory given by Hill [3]. The integral in (21) can then be expressed in a closed form, as observed already in [2], namely

$$
\begin{aligned}
k= & \sqrt{\frac{2}{1+R}}\left[\frac{n+1+2 R}{\sqrt{2(1+R)(1+2 R)}}\right]^{(n+1+2 R) /\left(n^{2}+1+2 R\right)} \\
& \cdot \exp \left[-\frac{(n-1) \sqrt{1+2 R}}{n^{2}+1+2 R} \arctan \frac{1}{\sqrt{1+2 R}}\right] .
\end{aligned}
$$

A further specification for the Mises material, with $R=1$, reads

$$
k=\left(\frac{n+3}{2 \sqrt{3}}\right)^{(n+3) /\left(n^{2}+3\right)} \exp \left[-\frac{\pi(n-1)}{2 \sqrt{3}\left(n^{2}+3\right)}\right] .
$$

Analogous expressions for the stress concentration at a circular hole (free boundary) are given in [7], [6], and [5].

For materials with $m=1$ the entire field is in an equibiaxial state of stress with the obvious result

$$
k=1 /(1+R) .
$$

The analogous expression for the hole problem [7] is

$$
k=\left(\frac{1}{1+R}\right)\left(\frac{n}{1+2 R}\right)^{(1+2 R) /(n-1-2 R)} .
$$

When $R=0$ we obtain from (24) and (25) the stress concentration factors for the standard Tresca material.

The constitutive relations for the rigid-ring problem when $m=1$ are not given by the usual normality rule since the whole field is at the corner regime of the yield locus. Instead we use the plastic work-equivalence relation, which leads here to the relation

$$
\varepsilon_{r}+\varepsilon_{\theta}=\frac{1}{1+R} \varepsilon
$$

Inserting (9) and (10) in (26) results in a differential equation for the radial displacement, with the solution

$$
u=\frac{\left(\sigma_{\infty} / \sigma_{0}\right)^{n}}{2(1+R)^{n+1}}\left(r-\frac{a^{2}}{r}\right)
$$




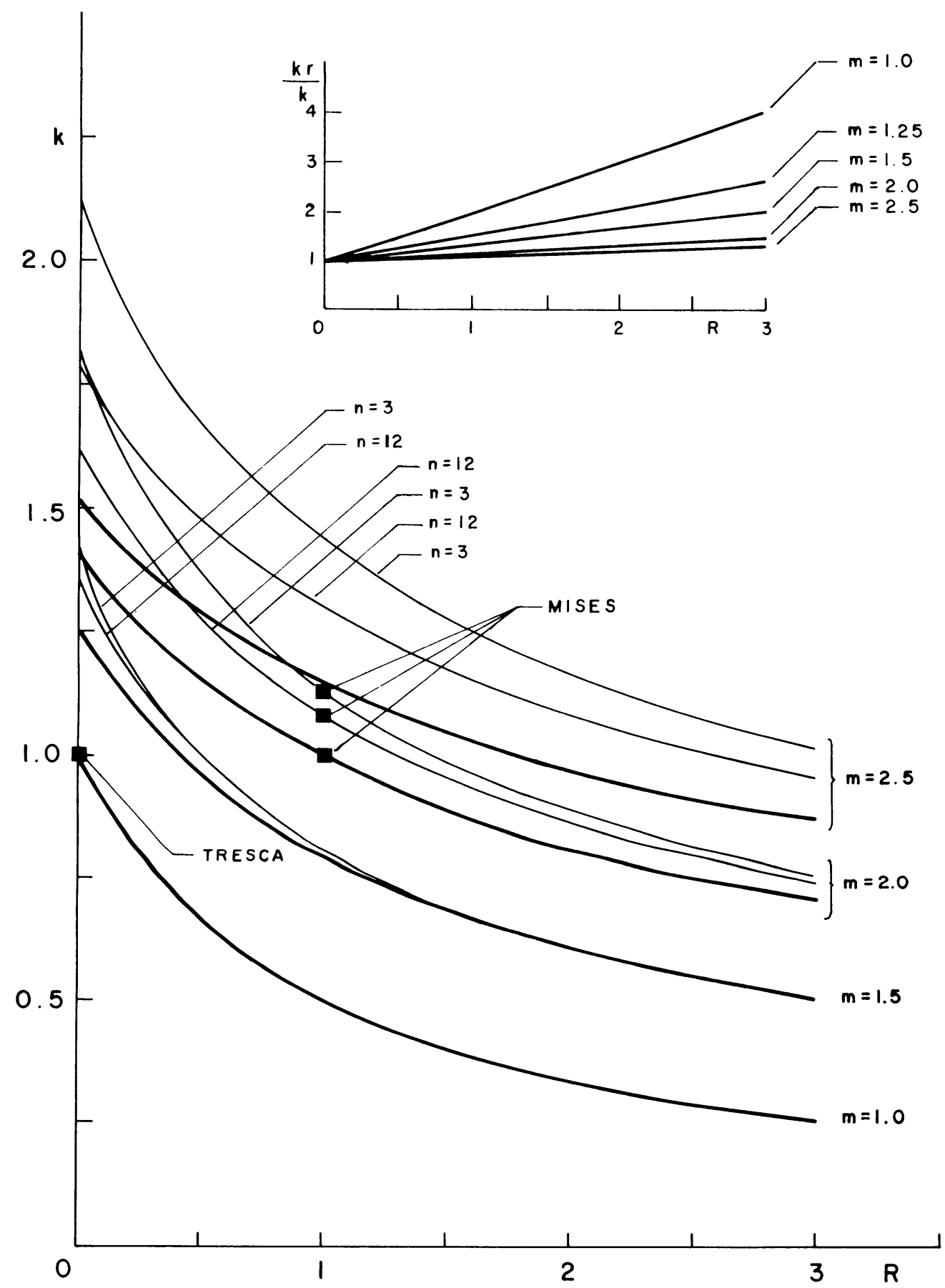

FIG. 3. Stress concentration factor for different values of the anisotropy parameters $(m, R)$ and hardening parameter $n$. Note the results for the Mises material $(m=2, R=1)$ and the Tresca material ( $m=1, R=0$ ). The heavy lines show the reference level $k=2 / H$ for a uniform sheet. The insert shows $k_{r} / k$ for different $m$ and $R$. 
It follows from (27) that the strain-rates ratio

$$
\dot{\varepsilon}_{\theta} / \dot{\varepsilon}_{r}=\left(r^{2}-a^{2}\right) /\left(r^{2}+a^{2}\right)
$$

remains bounded between 0 (at the hole) and 1 (at infinity).

Finally, we mention that the radial stress concentration factor defined as $k_{r}=\sigma_{r}(r=$ $a) / \sigma_{\infty}$ is equal to $S_{r}\left(\alpha_{a}\right) k$ or, with the aid of (6a) and (19),

$$
k_{r}=(J / 2)\left(1+I^{m /(m-1)}\right)^{(m-1) / m} k .
$$

Thus, the ratio $k_{r} / k$ is independent of $n$. That ratio is always greater than one (Fig. 3), so that $k_{r}>k$. With $m=1$ and $m=2$ we get the simple relations

$$
\left.\frac{k_{r}}{k}\right|_{m=1}=1+R,\left.\quad \frac{k_{r}}{k}\right|_{m=2}=\frac{1+R}{\sqrt{1+2 R}} .
$$

Acknowledgment. Thanks are due to the Technion-V. P. R. Fund-L. Rogow Fund for its financial support.

\section{REFERENCES}

[1] R. Hill, Theoretical plasticity of textured aggregates, Math. Proc. Cambridge Philos. Soc. 85, 179-191 (1979)

[2] W. H. Yang, Axisymmetric plane stress problems in anisotropic plasticity, J. Appl. Mech. 36, 7-14 (1969)

[3] R. Hill, A theory of the yielding and plastic flow of anisotropic metals, Proc. Roy. Soc. London Ser. A 193. 281-297 (1948)

[4] D. Durban, On two stress concentration problems in plane stress anisotropic plasticity, Internat. J. Solids and Structures. In press

[5] B. Budiansky and O. L. Mangasarian, Plastic stress concentration at a circular hole in an infinite sheet subjected to equal biaxial tension, J. Appl. Mech. 82, 59-64 (1960)

[6] B. Budiansky, An exact solution to an elastic-plastic stress concentration problem, PMM 35, 40-48 (1971)

[7] B. Budiansky, Anisotropic plasticity of plane-isotropic sheets, in Mechanics of Material Behaviour, pp. 15-29. Elsevier Science Publishers, Amsterdam, 1984

[8] D. Durban and V. Birman, On the elasto-plastic stress concentration at a circular hole in an anisotropic sheet, Acta Mech. 43, 73-84 (1982) 